\title{
Transformations to Speed the Convergence of Series ${ }^{1}$
}

\section{J. Barkley Rosser ${ }^{2}$}

\begin{abstract}
Numerical instances are given of the speeding of the convergence of series by the Euler transformation. This is even applied advantageously to certain divergent series, and a rigorous justification is given. An example is given of a series for which use of the Euler transformation is not useful. Instances are given of several less widely known methods. Finally, the method of summation by transformation into a continued fraction is illustrated successfully in the case of certain divergent series. The possibility of applying two different methods in succession to a given series is exploited throughout the paper, in spite of the fact that this often requires summing a divergent series.
\end{abstract}

A remarkably useful such transformation is based on a formula due to Euler:

$$
\sum_{n=0}^{\infty} a_{n} x^{n}=\frac{1}{1-x} \sum_{n=0}^{\infty}\left(\Delta^{n} a_{o}\right)\left(\frac{x}{1-x}\right)^{n} .
$$

We shall refer to this as Euler's transformation.

A purely formal derivation of this is as follows. Recalling that

$$
a_{n}=(1+\Delta)^{n} a_{o}
$$

we write

$$
\begin{aligned}
\sum_{n=0}^{\infty} a_{n} x^{n} & =\sum_{n=0}^{\infty} x^{n}(1+\Delta)^{n} a_{o}=\frac{1}{1-x(1+\Delta)} a_{o} \\
& =\frac{1}{1-x-x \Delta} a_{o}=\frac{1}{1-x} \frac{1}{1-\frac{x}{1-x} \Delta} a_{o} \\
& =\frac{1}{1-x} \sum_{n=0}^{\infty}\left(\frac{x}{1-x}\right)^{n} \Delta^{n} a_{o} .
\end{aligned}
$$

A rigorous derivation of eq 1 is given in $[1],^{3} \mathrm{pp}$. $62-66$. It is there stated that if the a's are all positive, then the right-hand side of eq 1 converges for every negative value of $x$ for which the left-hand side converges. That this is not necessarily true for positive $x$ is easily seen by taking $x=1$ and

$$
a_{n}=\frac{1}{(n+1)^{2}} .
$$

Actually, it is clear that the right side of eq 1 is not likely to be an improvement over the left side unless $x$ is negative and the $a$ 's are positive. In other words, the Euler Transformation might be expected to speed convergence only in the case of alternating series. However, for alternating series, the Euler Transformation is usually very helpful.

The Euler Transformation is a special case of a more general transformation given by Markoff (see [9], pp. 178-194). However, we confine our attention to the simpler Euler Transformation.

\footnotetext{
1 The preparation of this paper was sponsored (in part) by the Office of Naval Research.

${ }^{2}$ Help in carrying out the computations was furnished by Nancy Mann and Shirley Marks under the direction of Gertrude Blanch.

${ }^{3}$ Figures in brackets indicate the literature references at the end of this paper.
}

Applications of the Euler Transformation with $x=1$ give

$\log 2=\sum_{n=1}^{\infty} \frac{(-1)^{n-1}}{n}=\sum_{n=1}^{\infty} \frac{\mathbf{I}}{n 2^{n}}$,

$$
\frac{\pi}{4}=\arctan 1=\sum_{n=0}^{\infty} \frac{(-1)^{n}}{2 n+1}=\frac{1}{2} \sum_{n=0}^{\infty} \frac{n !}{1 \cdot 3 \cdot 5 \cdots(2 n+1)} .
$$

One recognizes the right side of eq 2 as the expansion of $-\log (1-1 / 2)$.

An interesting variation of the Euler Transformation is obtained as follows.

$$
\begin{aligned}
\sum_{n=0}^{\infty} a_{n} x^{n} & =\sum_{n=0}^{N-1} a_{n} x^{n}+x^{N} \sum_{m=0}^{\infty} a_{m+N} x^{m} \\
& =\sum_{n=0}^{N-1} a_{n} x^{n}+\frac{x^{N}}{1-x} \sum_{m=0}^{\infty}\left(\Delta^{m} a_{N}\right)\left(\frac{x}{1-x}\right)^{m}
\end{aligned}
$$

Applying this gives

$$
\begin{aligned}
\log (1+x) & =x \sum_{n=0}^{\infty} \frac{(-x)^{n}}{n+1}=x \sum_{n=0}^{N-1} \frac{(-x)^{n}}{n+1} \\
& +\frac{(-x)^{N}}{1+x} \sum_{m=0}^{\infty}\left(\frac{x}{1+x}\right)^{m} \frac{m !}{(N+1) \cdots(N+m+1)}
\end{aligned}
$$

Taking $N=8$ and $x=1$ gives

$\log 2=1-\frac{1}{2}+\frac{1}{3}-\frac{1}{4}+\frac{1}{5}-\frac{1}{6}+\frac{1}{7}-\frac{1}{8}$

$$
+\frac{1}{2}\left\{\frac{1}{9}+\frac{1}{2} \frac{1}{9 \cdot 10}+\frac{1}{4} \frac{1 \cdot 2}{9 \cdot 10 \cdot 11}+\frac{1}{8} \frac{1 \cdot 2 \cdot 3}{9 \cdot 10 \cdot 11 \cdot 12}+\cdots\right\}
$$

We get

$$
1-\frac{1}{2}+\cdots-\frac{1}{8}=0.63452381
$$

Also (see table 1), the terms inside the curly brackets add up to 0.11724674 , and we get

$\log 2=0.63452381+\frac{1}{2}(.011724674)=0.69314718$. 
TABLE 1

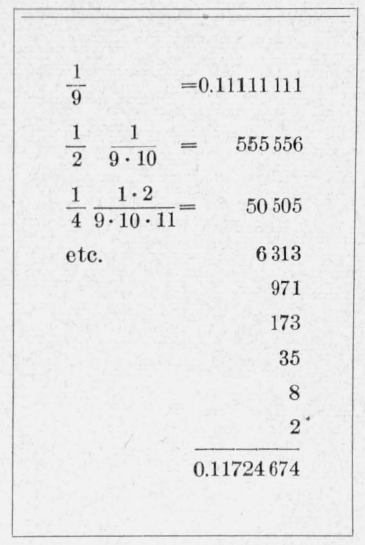

A more accurate value is $\log 2=0.6931471806$.

One of the great advantages of the Euler transformation is that it can easily be used for numerical computations even in cases where one has only numerical values of the coefficients to work with; indeed, the computations are very direct even in such cases, because only a simple differencing operation is called for.

A rather sensational use of the Euler transformation is in connection with asymptotic series. We give an instance.

We have for $y>0$

$$
\begin{aligned}
-y e^{y} E i(-y)= & \int_{0}^{\infty} \frac{e^{-t}}{1+\frac{t}{y}} d t \\
= & \int_{0}^{\infty} e^{-t}\left\{1-\frac{t}{y}+\frac{t^{2}}{y^{2}}-\frac{t^{3}}{y^{3}}+\cdots\right. \\
& \left.+\left(-\frac{t}{y}\right)^{N}+\left(-\frac{t}{y}\right)^{N+1} \frac{1}{1+\frac{t}{y}}\right\} d t \\
= & {\left[1-\frac{1 !}{y}+\frac{2 !}{y^{2}}-\frac{3 !}{y^{3}}+\cdots+\frac{N !}{(-y)^{N}}\right] } \\
+ & \int_{0}^{\infty}\left(-\frac{t}{y}\right)^{N+1} \frac{e^{-t}}{1+\frac{t}{y}} d t
\end{aligned}
$$

If the series within the square brackets were continued indefinitely, it would diverge strongly. Thus it does not appear that the Euler transformation is applicable in the present case. However, let us proceed formally to try to compute

$$
-5 e^{5} E i(-5)
$$

by use of the series alone. We get

$$
1-\frac{1}{5}+\frac{2}{25}-\frac{6}{125}+\cdots
$$

We have stopped just before the least term 24/625 and if we add up the four terms shown the error is less than the next term. That is, we get 0.83200 with an error less than 0.03840 . Comparing with a more accurate value, 0.85211 , we see that our error is indeed less than 0.03840 .

Formally, we have neglected the terms

$$
\frac{24}{625}-\frac{120}{3125}+\frac{720}{15625}-\ldots
$$

and properly so, since they begin to diverge rapidly. Nevertheless, let us now try to sum them by use of the Euler transformation, taking $x=-1$,

$$
\begin{aligned}
& a_{0}=\frac{24}{625} \\
& a_{1}=\frac{120}{3125} \\
& a_{2}=\frac{720}{15625} \\
& \text { etc. }
\end{aligned}
$$

We readily form the differences of the $a^{\prime}$ s numerically (see the appendix for some tricks for shortening the computation) and get

$$
\begin{aligned}
a_{0} & =0.0384000000 \\
\Delta a_{0} & =0.0000000000 \\
\Delta^{2} a_{0} & =0.0076800000 \\
\Delta^{3} a_{0} & =0.0030720000 \\
\Delta^{4} a_{0} & =0.0064512000 \\
\Delta^{5} a_{0} & =0.0076185600 \\
\Delta^{6} a_{0} & =0.0140697600 \\
\Delta^{7} a_{0} & =0.0260259840 \\
\Delta^{5} a_{0} & =0.0561340416 \\
\Delta^{9} a_{0} & =0.1314560410 \\
\Delta^{10} a_{0} & =0.3376621486 \\
\Delta^{11} a_{0} & =0.9382363791 \\
\Delta^{12} a_{0} & =2.8069767610 \\
\Delta^{13} a_{0} & =8.98851 \quad 15364 \\
\Delta^{14} a_{0} & =30.6682695734 \\
\Delta^{15} a_{0} & =111.0389871074
\end{aligned}
$$


So the Euler transformation, if applied formally, gives the series

$$
10^{-10}\{192000000+9600000-1920000+2016000
$$

$$
\begin{aligned}
& -1190400+1099200-1016640+1096368 \\
& -1283750+1648741-2290616+3426485 \\
& -5486152+9359213-16943205+\cdots \cdot\}
\end{aligned}
$$

This is actually a divergent series, and the last terms listed are already increasing. Nevertheless we treat it like an asymptotic series, and add up all terms preceding the one that is numerically least. This gives 0.0201604800 . Adding this to the 0.83200 that we had already, we get 0.8521604800 , which compares favorably with the more accurate value 0.85211 .

We neglected the terms

$$
-10^{-10}\{1016640-1096368+1283750-1648741
$$

$$
\begin{aligned}
& +2290616-3426485+5486152-9359213 \\
& +16943205-\cdots \cdot\}
\end{aligned}
$$

We now apply the Euler transformation to these terms, taking $x=-1$, and $a_{0}=1016640, a_{1}=1096368$, ... This gives

$$
\begin{aligned}
& -10^{-10}\{508320-19932+13457-4372+916-1383 \\
& +50-570+6 \ldots\}
\end{aligned}
$$

This adds up to -0.0000496486 if we stop before the least term. Adding this to our previous value gives 0.8521108314 , which compares very favorably with the more accurate value, 0.8521108814 .

The agreement with the more accurate value is experimental evidence that use of the Euler transformation is justifiable even in the present case where the series diverges. Actually, we can justify use of the Euler transformation rigorously for the present series. Looking at eq 4 , we see that the remainder can be written

$$
\left(-\frac{N}{y}\right)^{N+1} \frac{1}{2} \int_{0}^{\infty}\left(\frac{t}{N}\right)^{N+1} \frac{e^{-t}}{1+\frac{1}{2}\left(\frac{t}{y}-1\right)} d t
$$

which can be expanded as

$$
\begin{aligned}
& \left(-\frac{N}{y}\right)^{N+1}\left\{\frac{1}{2} \int_{0}^{\infty}\left(\frac{t}{N}\right)^{N+1} e^{-t} d t\right. \\
& -\frac{1}{4} \int_{0}^{\infty}\left(\frac{t}{N}\right)^{N+1}\left(\frac{t}{y}-1\right) e^{-t} d t \\
& +\frac{1}{8} \int_{0}^{\infty}\left(\frac{t}{N}\right)^{N+1}\left(\frac{t}{y}-1\right)^{2} e^{-t} d t-\ldots
\end{aligned}
$$

$$
\begin{aligned}
& +\frac{(-1)^{M}}{2^{M+1}} \int_{0}^{\infty}\left(\frac{t}{N}\right)^{N+1}\left(\frac{t}{y}-1\right)^{M} e^{-t} d t \\
& +\frac{(-1)^{M+1}}{2^{M+2}} \int_{0}^{\infty}\left(\frac{t}{N}\right)^{N+1}\left(\frac{t}{y}-1\right)^{M+1} \frac{e^{-t}}{1+\frac{1}{2}\left(\frac{t}{y}-1\right)} d t
\end{aligned}
$$

If we put $N=3$ and $y=5$, we easily verify that

$$
\int_{0}^{\infty}\left(\frac{t}{N}\right)^{N+1}\left(\frac{t}{y}-1\right)^{M} e^{-t} d t=\Delta^{M} a_{0} .
$$

We then see that all differences of $a_{0}$ of even order are positive, and that if we stop with a term involving a difference of odd order, the error involved is less than the next term (which involves a positive difference of even order).

To justify a second application of the Euler transformation, we modify the remainder term given above as follows:

$$
\begin{aligned}
\int_{0}^{\infty}\left(\frac{t}{N}\right)^{N+1}\left(\frac{t}{y}-1\right)^{M+1} \frac{e^{-t}}{1+\frac{1}{2}\left(\frac{t}{y}-1\right)} d t \\
\quad=\frac{1}{2} \int_{0}^{\infty}\left(\frac{t}{N}\right)^{N+1}\left(\frac{t}{y}-1\right)^{M+1} \frac{e^{-t} d t}{1+\frac{1}{2}\left(\frac{1}{2}\left(\frac{t}{y}-1\right)-1\right)} \\
=\frac{1}{2} \int_{0}^{\infty}\left(\frac{t}{N}\right)^{N+1}\left(\frac{t}{y}-1\right)^{M+1} e^{-t} d t \\
\quad-\frac{1}{4} \int_{0}^{\infty}\left(\frac{t}{N}\right)^{N+1}\left(\frac{t}{y}-1\right)^{M+1}\left(\frac{1}{2}\left(\frac{t}{y}-1\right)-1\right) e^{-t} d t+\cdots
\end{aligned}
$$

We can apply the Euler transformation to other asymtotic series with equal success. For instance we have

$$
\begin{gathered}
2 w e^{w^{2}} \int_{w}^{\infty} e^{-x^{2}} d x=1-\frac{1}{2 w^{2}}+\frac{1 \cdot 3}{\left(2 w^{2}\right)^{2}}-\frac{1 \cdot 3 \cdot 5}{\left(2 w^{2}\right)^{3}} \\
+\frac{1 \cdot 3 \cdot 5 \cdot 7}{\left(2 w^{2}\right)^{4}}+\ldots+\frac{1 \cdot 3 \cdot 5 \ldots(2 N-1)}{\left(-2 w^{2}\right)^{N}} \\
+\frac{2}{\sqrt{\pi}\left(-w^{2}\right)^{N+1}} \int_{0}^{\infty} \frac{y^{2 N+2} e^{-y^{2}}}{1+\frac{y^{2}}{w^{2}}} d y
\end{gathered}
$$

If we now write

$$
\int_{0}^{\infty} \frac{y^{2 N+2} e^{-y^{2}}}{1+\frac{y^{2}}{w^{2}}} d y=\frac{1}{2} \int_{0}^{\infty} \frac{y^{2 N+2} e^{-y^{2}}}{1+\frac{1}{2}\left(\frac{y^{2}}{w^{2}}-1\right)} d y
$$

and expand

$$
\frac{1}{1+\frac{1}{2}\left(\frac{y^{2}}{w^{2}}-1\right)}
$$


we can justify applying the Euler transformation to the asymptotic series

$$
1-\frac{1}{2 w^{2}}+\frac{1 \cdot 3}{\left(2 w^{2}\right)^{2}}-\ldots
$$

We applied the Euler transformation to this series with $w=6$ to compute a 20 decimal value of

$$
12 e^{36} \int_{6}^{\infty} e^{-x^{2}} d x
$$

which was then checked by a different means of computation (see [3], p. 86).

We can use the Euler transformation to derive Airey's "Converging Factor" (see [2]). In those cases (as above) in which we can justify using the Euler transformation on an asymptotic series, we are then afforded a means of justifying the use of Airey's "Converging Factor."

To see how this is done, let us write.

$$
\begin{aligned}
-y e^{y} E i(-y)=1 & -\frac{1 !}{y}+\frac{2 !}{y^{2}}-\frac{3 !}{y^{3}}+\cdots+\frac{(N-2) !}{(-y)^{N-2}} \\
& +\frac{(N-1) !}{(-y)^{N-1}}\left\{1-\frac{N}{y}+\frac{N(N+1)}{y^{2}}\right. \\
& \left.-\frac{N(N+1)(N+2)}{y^{3}}+\cdots\right\} .
\end{aligned}
$$

Airey refers to the series in the curly brackets as the "Converging Factor" and gives a purely formal device for estimating a value of it (Airey's estimate is justified in [12]). We will now show how to derive Airey's formulas by use of the Euler transformation, which we have already shown to be justified for the present series. We write

$$
y=\frac{\nu}{\beta}, \nu=N+h .
$$

Then the "Converging Factor" takes the form

$$
1-\left(1-\frac{h}{\nu}\right) \beta+\left(1-\frac{h}{\nu}\right)\left(1+\frac{1-h}{\nu}\right) \beta^{2}-\cdots
$$

We now apply the Euler transformation with

$$
\begin{aligned}
& x=-\beta, \\
& a_{o}=1 \\
& a_{1}=\left(1-\frac{h}{\nu}\right) \\
& a_{2}=\left(1-\frac{h}{\nu}\right)\left(1+\frac{1-h}{\nu}\right)
\end{aligned}
$$

etc.
We find

$$
\begin{aligned}
a_{o} & =1 \\
\Delta a_{0} & =-\frac{h}{\nu} \\
\Delta^{2} a_{o} & =\frac{1}{\nu}-\frac{h(1-h)}{\nu^{2}} \\
\Delta^{3} a_{o} & =\frac{2-3 h}{\nu^{2}}-\frac{h(1-h)(2-h)}{\nu^{3}}
\end{aligned}
$$

etc.

So use of the Euler transformation gives us

$$
\begin{aligned}
& \frac{1}{1+\beta}\left\{1+\frac{\beta}{1+\beta} \frac{h}{\nu}+\left(\frac{\beta}{1+\beta}\right)^{2}\left(\frac{1}{\nu}-\frac{h(1-h)}{\nu^{2}}\right)\right. \\
& \left.+\left(\frac{\beta}{1+\beta}\right)^{3}\left(\frac{2-3 h}{\nu^{2}}-\frac{h(1-h)(2-h)}{\nu^{3}}\right)+\cdots\right\}
\end{aligned}
$$

If we rearrange this in powers of $1 / \nu$ (such a rearrangement needs justification), we find that it is identical with the formula on p. 526 of [2].

If we choose $\nu=N, y=N$, so that $h=0, \beta=1$, the "Converging Factor" reduces to

$$
\frac{1}{2}\left(1+\sum_{n=1} \frac{C_{n}}{(4 N)^{n}}\right)
$$

with $C_{1}=1, C_{2}=-1, C_{3}=-1, C_{4}=13$, etc. The values of the first $22 C^{\prime}$ so are given on p. 529 of [2]. Using the first 17 of these with $N=5$ we get the following series for the "Converging Factor":

$$
\begin{aligned}
& \frac{1}{2} 10^{-15}\{1000000000000000+50000000000000 \\
& -2500000000000-125000000000+81250000000 \\
& -14687500000-1140625000+1911718750 \\
& -656679688-30373047+168604590-92748286 \\
& +2680585+36418569-29553896+4119945 \\
& +15450012-17702724+\ldots\} .
\end{aligned}
$$

This series is probably divergent if carried far enough, but we sum it as if it were an asymptotic series and stop just before the least term shown. This gives the value 0.52372086 . Airey gets 0.52372087 (see [2] p. 529), but the difference of 1 unit in the last place is likely due to differences in rounding procedure. This should be multiplied by 0.0384 and added to 0.832 , which gives 0.8521108810 as compared with the more accurate value 0.8521108814 .

In [2], Airey applies the "Converging Factor" to various asymptotic series without rigorous justifica- 
tion, but with great numerical success. By deriving the "Converging Factor" by means of the Euler transformation, one could justify its use in some of the cases given in [2], though there would still remain the problem of justifying various rearrangements made by Airey of the series in the "Converging Factor." Some of these can be justified by the procedure in [12].

Although we have seen that the Euler transformation can be applied in some rather remarkable situations, nevertheless there are cases where it cannot be applied. In particular, for nonalternating series (for instance, when $x$ and the $a$ 's are all positive in (1)), it is of almost no use. However even for alternating series it occasionally fails, and we will now give an alternating series for which it is of no value whatever.

Let us expand

$$
\int_{0}^{1} \frac{d x}{1+x^{2}}
$$

by the Euler-Maclaurin sum formula (see [4], p. 128) using an interval of length unity. We first need the values of the derivatives of

$$
\frac{1}{1+x^{2}}
$$

of odd order evaluated at 0 and 1 . At 0 , the derivatives of odd order are zero. To get the values at 1 we write

$$
\begin{aligned}
\frac{1}{1+x^{2}} & =\frac{1}{2 i}\left(\frac{1}{x-i}-\frac{1}{x+i}\right) \\
\frac{d^{n}}{d x^{n}} \frac{1}{1+x^{2}} & =\frac{1}{2 i}\left(\frac{(-1)^{n} n !}{(x-i)^{n+1}}-\frac{(-1)^{n} n !}{(x+i)^{n+1}}\right) \\
& =\frac{(-1)^{n} n !}{2 i\left(1+x^{2}\right)^{n+1}}\left\{(x+i)^{n+1}-(x-i)^{n+1}\right\}
\end{aligned}
$$

Putting $x=1$ and recalling

$$
\begin{aligned}
& 1+i=\sqrt{2} e^{\frac{\pi i}{4}} \\
& 1-i=\sqrt{2} e^{-\frac{\pi i}{4}}
\end{aligned}
$$

we get

$$
\begin{aligned}
{\left[\frac{d^{n}}{d x^{n}} \frac{1}{1+x^{2}}\right]_{x=1} } & =\frac{(-1)^{n} n !}{2 i(\sqrt{2})^{n+1}}\left\{e^{\frac{(n+1) \pi i}{4}}-e^{-\frac{(n+1) \pi i}{4}}\right\} \\
& =\frac{(-1)^{n} n !}{(\sqrt{2})^{n+1}} \sin \frac{(n+1) \pi}{4}
\end{aligned}
$$

So

$$
\left[\frac{d^{2 m-1}}{d x^{2 m-1}} \frac{1}{1+x^{2}}\right]_{x=1}=-\frac{(2 m-1) !}{2^{m}} \sin \frac{\pi m}{2} .
$$

Substituting in the formula

$$
\begin{aligned}
\int_{0}^{1} F(x) d x & =\frac{1}{2} F(0)+\frac{1}{2} F(1) \\
& +\sum_{m=1}^{n-1} \frac{(-1)^{m} B_{m}}{(2 m) !}\left\{F^{(2 m-1)}(1)-F^{(2 m-1)}(0)\right\}+R_{n}
\end{aligned}
$$

(see [4], p. 128, with $\omega=1, r=1$ ) we get

$$
\begin{aligned}
\int_{0}^{1} \frac{d x}{1+x^{2}} & =\frac{3}{4}-\sum_{m=0}^{n-1} \frac{(-1)^{m} B_{m}}{m 2^{m+1}} \sin \frac{\pi m}{2}+R_{n} \\
& =\frac{3}{4}+\sum_{r=0}^{s-1} \frac{B_{2 r+1}(-1)^{r}}{(2 r+1) 4^{r+1}}+R_{2 s} \\
& =\frac{3}{4}+\frac{1}{24}-\frac{1}{2016}+\frac{1}{4224}-\frac{1}{1536} \\
& +\frac{43867}{7354368}-\frac{77683}{565248}+\frac{657931}{98304}-\cdots+R .
\end{aligned}
$$

This is certainly an alternating series. However, the partial sums do not lie alternately above and below the true value. In table 2 we have given the terms of the series with the partial sums to the right. If we try to improve the value by use of the Euler transformation, we get $10^{-5}(79117+\{12-10+61-$ $758+. \cdot\}$ ), which is certainly no better.

TABLE 2.

\begin{tabular}{|r|r|}
\hline \multicolumn{1}{|c|}{ Series } & Partial sums \\
\hline+0.75000 & 0.75000 \\
+4167 & .79167 \\
-50 & .79117 \\
+24 & .79141 \\
-65 & .79076 \\
+596 & .79672 \\
-0.13743 & .65929 \\
+6.69282 & 7.35211 \\
\hline \multicolumn{2}{|c|}{ True value _... 0.7854} \\
\hline
\end{tabular}

As indicated, the Euler transformation is valueless for speeding convergence of a nonalternating series. Occasionally one can use a trick to transform a nonalternating series to an alternating series. Thus the formula

$$
\sum_{n=1}^{\infty} \frac{1}{n^{2}}=2 \sum_{n=1}^{\infty} \frac{(-1)^{n+1}}{n^{2}}
$$

enables us to get a value for

$$
\sum_{n=1}^{\infty} \frac{1}{n^{2}}
$$

by (indirect) use of the Euler transformation. However, in general such tricks are not available.

Fortunately the Euler-Maclaurin sum formula is of great value for summing many nonalternating series. For instance, by use of the Euler-MacLaurin sum formula (see [4], p. 128) we prove that for $m \geq 1, s>1$, 


$$
\begin{aligned}
\sum_{n=1}^{\infty} \frac{1}{n^{s}}= & \sum_{n=1}^{m} \frac{1}{n^{s}}+\frac{m^{1-s}}{s-1}-\frac{m^{-s}}{2} \\
& -\sum_{r=1} \frac{s(s+1) \ldots(s+2 r-2)}{(2 r) !} \frac{(-1)^{r} B_{r}}{m^{2 r+s-1}} .
\end{aligned}
$$

The series on the right is divergent, but it is an asymptotic series, and by taking $m$ large compared to $s$, considerable accuracy can be obtained, which can be further increased by applying the Euler transformation to the asymptotic series. Thus, consider the case where $s=2$. We have the well known result

$$
\sum_{n=1}^{\infty} \frac{1}{n^{2}}=\frac{\pi^{2}}{6}=1.6449340668 .
$$

Even with $m$ as small as 2 we get

$$
\begin{gathered}
1+\frac{1}{4}+\frac{1}{2}-\frac{1}{8}+\frac{1}{48}-\frac{1}{960}+\frac{1}{5376}-\frac{1}{15360}+\frac{5}{135168} \\
-\frac{691}{22364160}+\frac{7}{196608}-\frac{3617}{66846720}+\frac{43867}{418381824} \\
-\frac{174611}{692060160}+\frac{854513}{1157627904}-\ldots
\end{gathered}
$$

This is divergent, but if we sum all terms before the least, which is

$$
\frac{691}{22364160}
$$

we get 1.64495 as compared to the more accurate value 1.64493 . If we now apply the Euler transformation to the terms neglected, we get the series

$$
\begin{aligned}
& -10^{-10}\{154488-11765+17249-11523 \\
& +14389-20493+\ldots\} .
\end{aligned}
$$

Doubtless this diverges, but if we sum all terms before the least, we get 1.6449336 as compared with the more accurate value 1.64493 41. Another use of the Euler transformation on the terms neglected above gives further improvement.

In [5], Bickley and Miller give a method for dealing with series $\Sigma a_{n}$ in which

$$
\frac{a_{n}}{a_{n-1}}=1-\frac{A_{1}}{n}+\frac{A_{2}}{n^{2}}+\frac{A_{3}}{n^{3}}+\cdots
$$

In those cases in which $a_{n}$ is a differentiable function of $n$, one can treat such series by the EulerMaclaurin sum formula, and usually one gets the same approximation as would be given by the Bickley-Miller method.

In the Bickley-Miller method, one writes

$$
\begin{gathered}
S=\sum_{n=0}^{\infty} a_{n} \\
S_{m}=\sum_{n=0}^{m} a_{n} .
\end{gathered}
$$

Then one tries to determine constants $\alpha_{-1}, \alpha_{0}, \alpha_{1}$, . . so that

$$
S-S_{m}=a_{m}\left\{\alpha_{-1} m+\alpha_{0}+\frac{\alpha_{1}}{m}+\frac{\alpha_{2}}{m^{2}}+\cdots\right\} .
$$

In general, this is impossible, since the series

$$
\alpha_{-1} m+\alpha_{0}+\frac{\alpha_{1}}{m}+\cdots
$$

will be divergent for each $m$. However, usually this series is an asymptotic series, so that for large $m$ great accuracy is attainable.

To determine the $\alpha$ 's, we proceed formally as follows. By eq 7 ,

$$
\begin{aligned}
S-S_{m-1}= & a_{m-1}\left\{\alpha_{-1}(m-1)+\alpha_{0}+\frac{\alpha_{1}}{m-1}\right. \\
& \left.+\frac{\alpha_{2}}{(m-1)^{2}}+\cdots\right\}
\end{aligned}
$$

Subtracting eq 7 from this gives

$$
a_{m}=S_{m}-S_{m-1}=a_{m-1}\{\}-a_{m}\{\} .
$$

However, by eq 6 ,

$$
a_{m}=a_{m-1}\left(1-\frac{A_{1}}{m}+\frac{A_{2}}{m^{2}}+\cdots\right) .
$$

Substituting this and factoring out $a_{m-1}$ and dividing by $m$ gives

$$
\begin{aligned}
\frac{1}{m}-\frac{A_{1}}{m^{2}}+\frac{A_{2}}{m^{3}}+\cdots=\alpha_{-1}-\frac{\alpha_{-1}}{m}+\frac{\alpha_{0}}{m}+\frac{\alpha_{1}}{m(m-1)} \\
\quad+\cdots-\left(1-\frac{A_{1}}{m}+\frac{A_{2}}{m^{2}}+\cdots\right)\left\{\alpha_{-1}+\frac{\alpha_{0}}{m}+\cdots\right\}
\end{aligned}
$$

If we now expand

$$
\frac{1}{m(m-1)^{r}}=\frac{1}{m^{r+1}}+\frac{r}{1 ! m^{r+2}}+\frac{r(r+1)}{2 ! m^{r+3}}+\cdots
$$

and equate powers of $\frac{1}{m}$, we can determine the $\alpha$ 's. Indeed we have

$$
\begin{aligned}
& \alpha_{-1}\left(A_{1}-1\right)=1, \\
& \left(\alpha_{0}+1\right) A_{1}=\alpha_{-1} A_{2}, \\
& \alpha_{1}\left(A_{1}+1\right)=\left(\alpha_{0}+1\right) A_{2}+\alpha_{-1} A_{3},
\end{aligned}
$$

etc.

(Note error in sign in formula (12c) on p. 757 of [5]).

If we apply the Bickley-Miller method to the series

$$
\sum_{n=1}^{\infty} \frac{1}{n^{s}}
$$


we get the same expansion that is given by the EulerMaclaurin sum formula (see above). Bickley and Miller do not apply their method to any series of interest that cannot be handled by the Euler-Maclaurin sum formula, but they indicate how such series might arise. Bickley and Miller do not give a rigorous justification of their method, but Szász (in [10]) has discussed the conditions under which one can use at least the term $\alpha_{-1} m$ in eq 7 for a first approximation to $S$. It would be very helpful to have a general treatment of the conditions under which the BickleyMiller method can be used.

In [7] and [8], Stieltjes gives some interesting ways for estimating the error in certain well known asymptotic series. However, his methods are rather special, and can hardly be applied to series at random.

We turn now to what is perhaps the most widely applicable method that we know. This is the method of transforming the series into a continued fraction.

Let us return to the example given above in which we got an approximation for

$$
\sum_{n=1}^{\infty} \frac{1}{n^{2}}
$$

by the Euler-Maclaurin sum formula. We had a divergent series, of which we neglected all terms beginning with the least. Let us now try to find a sum for these neglected terms by transforming them into a continued fraction. To write them as a continued fraction, we first consider the series

$$
\begin{gathered}
-\left\{\frac{691}{22364160} \frac{1}{z}-\frac{7}{196608} \frac{1}{z^{3}}+\frac{3617}{66846720} \frac{1}{z^{5}}\right. \\
-\frac{43867}{418381824} \frac{1}{z^{7}}+\frac{174611}{692060160} \frac{1}{z^{9}} \\
\left.-\frac{854513}{1157627904} \frac{1}{z^{11}}+\cdots\right\} .
\end{gathered}
$$

Transforming this into a continued fraction by the procedure given on pp. 196 to 202 of [6] we get

$$
\frac{a_{0}}{z}+\frac{a_{1}}{z}+\frac{a_{2}}{z}+\frac{a_{3}}{z}+\cdots
$$

with the coefficients

$$
\begin{aligned}
& a_{0}=0.00003089765053 \\
& a_{1}=1.152315485 \\
& a_{2}=0.367432414 \\
& a_{3}=1.728891714 \\
& a_{4}=0.861552894 \\
& a_{5}=2.427331997
\end{aligned}
$$

(Our $a$ 's after $a_{0}$ are taken to be the negatives of those defined in [6]. Due to a gradual loss of significant figures, the final digits of our $a$ 's are increasingly inaccurate.)

We then put $z=1$, and use the resulting continued fraction to compute an estimate for our remainder (which, by subtraction from the known sum of the series should come out to be -0.0000154985$)$. The successive convergents are:

$$
\begin{aligned}
& -0.0000308977 \\
& -0.0000143555 \\
& -0.0000167677 \\
& -0.0000153294 \\
& -0.0000157006 \\
& -0.0000154620
\end{aligned}
$$

We note the characteristic continued fraction behavior of the convergents in that they are successively above and below the true value, and monotonely approach the true value from above and below. Already the sixth convergent gives a value correct within less than 4 in the eighth decimal place, and it seems very plausible to conjecture that later convergents will continue to come ever closer, in spite of the fact that it is a divergent series that we are transforming into a continued fraction. This is a not uncommon phenomenon with continued fractions; in fact this is the basis of the Stieltjes method for summing asymptotic series. In [6] on pp. 349 to $361, p$. 365, p. 367 , and pp. 372 to 373 are given many examples of convergent continued fractions, which are the formal equivalents of divergent asymptotic series, including some that resemble very closely the series with which we are dealing.

Note that we used only odd powers of $z$ in our series

$$
-\left\{\frac{691}{22364160} \frac{1}{z}-\ldots\right\} \text {. }
$$

This has the effect of greatly reducing the labor of finding the corresponding continued fraction (in the notation of pp. 196 to 202 of [6], all the $b$ 's will be zero).

For an additional instance of the use of continued fractions, let us recall the series for the "Converging Factor" which we had earlier. We used all terms preceding the least, leaving several terms unused. Let us now try to attach a value to these unused terms by transforming them to a continued fraction. To avoid difficulties with the continued fraction expansion, we start with the term after the least. We formally transform the series

$$
\begin{array}{r}
\frac{1}{2} 10^{-15}\left\{\frac{36418569}{z}-\frac{29553896}{z^{3}}+\frac{4119945}{z^{5}}\right. \\
\left.+\frac{15450012}{z^{7}}-\frac{17702724}{z^{9}}+\cdots\right\}
\end{array}
$$


into a continued fraction

$$
\frac{a_{0}}{z}+\frac{a_{1}}{z}+\cdots
$$

getting

$$
\begin{aligned}
& a_{0}=0.000000018209284 \\
& a_{1}=0.81150624 \\
& a_{2}=-0.67210178 \\
& a_{3}=0.80673455 \\
& a_{4}=0.02419193 .
\end{aligned}
$$

Because one of the $a$ 's is negative, we no longer get the familiar performance of the convergents. Nevertheless, they are apparently converging to the true value 0.000000007869 since they are

$$
\begin{aligned}
& 0.000000018209 \\
& 0.000000010052 \\
& 0.000000005240 \\
& 0.000000007944 \\
& 0.0000000079 \quad 16
\end{aligned}
$$

respectively. Using the last, we get a value of 0.852110881425 for $-5 e^{5} \mathrm{Ei}(-5)$ as compared with the more accurate value 0.8521108814237 .

One other remarkable use of continued fractions is given in [11], where the asymptotic series

$e^{-w^{2}} \int_{0}^{w} e y^{2} d y=\frac{1}{2 w}+\frac{1}{4 w^{3}}+\frac{1 \cdot 3}{8 w^{5}}+\frac{1 \cdot 3 \cdot 5}{16 w^{7}}+\frac{1 \cdot 3 \cdot 5 \cdot 7}{32 w^{9}}+\cdots$ is converted into the continued fraction

$$
\frac{1}{2 w}-\frac{2}{2 w}-\frac{4}{2 w}-\frac{6}{2 w}-\frac{8}{2 w}-\cdots
$$

This continued fraction was used to compute portions of a table (see Section 26 of [11]) in spite of the fact that the continued fraction is likely divergent due to the fact that occasional convergents will have extremely small denominators. However, as shown by the estimates in Section 8 of [11], whenever we have a convergent with a large denominator, this convergent is a close approximation to the value of the function.

In closing, we might remark that some of the transformations studied in theories of summability are occasionally useful to speed convergence. Thus the use of Riesz sums,

$$
\sigma_{n}=\frac{\sum_{m=1}^{n} \lambda_{m} S_{m}}{\sum_{m=1}^{n} \lambda_{m}},
$$

where the $\lambda_{m}$ are increasing positive numbers, is sometimes helpful in estimating the sum of an alternating series of which $S_{m}$ are the partial sums.

\section{Appendix. Methods of shortening the computation of $\Delta^{m} a_{0}$.}

In an earlier draft of this appendix, we set forth a scheme for shortening the computation of $\Delta^{m} a_{0}$. Upon reading this earlier draft, Prof. J. C. P. Miller proposed the following very ingenious scheme for computing $\Delta^{m} a_{0}$.

In the text, we put

$$
\begin{aligned}
& a_{0}=\frac{24}{625}, \\
& a_{1}=\frac{120}{3125}, \\
& a_{2}=\frac{720}{15625}, \\
& \text { etc. }
\end{aligned}
$$

and wished to compute $\Delta^{m} a_{0}$. To do this more quickly, we write

$$
a_{n}=\frac{(N-1) !}{(-N)^{N-1}} \alpha_{n}
$$

where $N=5$ and

$$
\begin{aligned}
& \alpha_{0}=1, \\
& \alpha_{1}=1 \\
& \alpha_{2}=1+\frac{1}{N}, \\
& \alpha_{3}=\left(1+\frac{1}{N}\right)\left(1+\frac{2}{N}\right), \\
& \alpha_{4}=\left(1+\frac{1}{N}\right)\left(1+\frac{2}{N}\right)\left(1+\frac{3}{N}\right),
\end{aligned}
$$

etc.

Then

$$
\Delta^{m} a_{n}=\frac{(N-1) !}{(-N)^{N-1}} \Delta^{m} \alpha_{n} .
$$

So we seek a formula for $\Delta^{m} \alpha_{n}$.

Actual subtraction gives

$$
\Delta \alpha_{n}=\frac{n}{N} \alpha_{n} .
$$


Then we prove by induction on $m$ that

$$
\Delta^{m+1} \alpha_{n}=\frac{n+m}{N} \Delta^{m} \alpha_{n}+\frac{m}{N} \Delta^{m-1} \alpha_{n} .
$$

Putting $n=0$ gives

$$
\Delta^{m+1} \alpha_{0}=\frac{m}{N}\left(\Delta^{m} \alpha_{0}+\Delta^{m-1} \alpha_{0}\right) .
$$

Then

$$
\Delta^{m+1} a_{0}=\frac{m}{N}\left(\Delta^{m} a_{0}+\Delta^{m-1} a_{0}\right) .
$$

Recalling that it is actually

$$
\frac{\Delta^{m} a_{0}}{2^{m+1}}
$$

that we seek, we write the simple recursion

$$
\frac{\Delta^{m+1} a_{0}}{2^{m+2}}=\frac{m}{4 N}\left(2 \frac{\Delta^{m} a_{0}}{2^{m+1}}+\frac{\Delta^{m-1} a_{0}}{2^{m}}\right) .
$$

Using this, we readily compute numerical values of

$$
\frac{\Delta^{m} a_{0}}{2^{m+1}}
$$

In our earlier scheme, we had expressed $\Delta^{m} \alpha_{0}$ as a polynomial in $(1 / N)$, and listed the following results (which can easily be verified by use of eq A):

$$
\begin{aligned}
\Delta \alpha_{0} & =0 \\
\Delta^{2} \alpha_{0} & =\frac{1}{N^{2}} \\
\Delta^{3} \alpha_{0} & =\frac{2}{N^{2}}, \\
\Delta^{4} \alpha_{0} & =\frac{6}{N^{3}}+\frac{3}{N^{2}}, \\
\Delta^{5} \alpha_{0} & =\frac{24}{N^{4}}+\frac{20}{N^{3}}, \\
\Delta^{6} \alpha_{0} & =\frac{120}{N^{5}}+\frac{130}{N^{4}}+\frac{15}{N^{3}}, \\
\Delta^{7} \alpha_{0} & =\frac{720}{N^{6}}+\frac{924}{N^{5}}+\frac{210}{N^{4}}, \\
\Delta^{8} \alpha_{0} & =\frac{5040}{N^{7}}+\frac{7308}{N^{6}}+\frac{2380}{N^{5}}+\frac{105}{N^{4}},
\end{aligned}
$$

$\Delta^{9} \alpha_{0}=\frac{40320}{N^{8}}+\frac{64224}{N^{7}}+\frac{26432}{N^{6}}+\frac{2520}{N^{5}}$

$\Delta^{10} \alpha_{0}=\frac{362880}{N^{9}}+\frac{623376}{N^{8}}+\frac{303660}{N^{7}}+\frac{44100}{N^{6}}+\frac{945}{N^{5}}$,

$\Delta^{11} \alpha_{0}=\frac{3628800}{N^{10}}+\frac{6636960}{N^{9}}+\frac{3678840}{N^{8}}+\frac{705320}{N^{7}}+\frac{34650}{N^{6}}$,

$\Delta^{12} \alpha_{0}=\frac{39916800}{N^{11}}+\frac{76998240}{N^{10}}+\frac{47324376}{N^{9}}+\frac{11098780}{N^{8}}$

$+\frac{866250}{N^{7}}+\frac{10395}{N^{6}}$

$\Delta^{13} \alpha_{0}=\frac{479001600}{N^{12}}+\frac{967524480}{N^{11}}+\frac{647536032}{N^{10}}$

$$
+\frac{177331440}{N^{9}}+\frac{18858840}{N^{8}}+\frac{540540}{N^{7}}
$$

$\Delta^{14} \alpha_{0}=\frac{6227020800}{N^{13}}+\frac{13096736640}{N^{12}}+\frac{9418945536}{N^{11}}$

$$
+\frac{2920525608}{N^{10}}+\frac{389449060}{N^{9}}+\frac{18288270}{N^{8}}+\frac{135135}{N^{7}},
$$

$\Delta^{15} \alpha_{0}=\frac{87178291200}{N^{14}}+\frac{190060335360}{N^{13}}+\frac{145410580224}{N^{12}}$

$+\frac{49952862960}{N^{11}}+\frac{7934927000}{N^{10}}+\frac{520059540}{N^{9}}+\frac{9459450}{N^{8}}$.

\section{References}

[1] T. J. I'a. Bromwich, An Introduction to the theory of infinite series (Macmillan and Co., London, 1947, 2d ed. revised).

[2] John R. Airey, The "Converging Factor" in asymptotic series and the calculation of Bessel, Laguerre and other functions, Phil. Mag. [7] 24, 521 to 552 (1937).

[3] Mathematical tables and other aids to computation, ? (1946-47).

[4] E. T. Whittaker and G. N. Watson, Modern analysis (The Macmillan Co., 1947, American ed.).

[5] W. G. Bickley and J. C. P. Miller, The numerical summation of slowly convergent series of positive terms, Phil. Mag. [7] 22, 754 to 767 (1936).

[6] H. S. Wall, Analytic theory of continued fractions (D. van Nostrand Co., Ine., New York, N. Y., 1948).

[7] Th. Stieltjes, Recherches sur quelques séries semiconvergentes, Ann. Sci. de L'Ecole Normale Supérieure [3] 3, 201 to 258 (1886).

[8] T. J. Stieltjes, Note sur un développement de l'intégrale $\int_{0}^{a} e^{x^{2}} d x$, Acta Math. 9, 167 to 176 (1886).

[9] A. A. Markoff, Differenzenrechnung, Teubner (1896). (Translated by Th. Friesendorff and E. Prümm).

[10] O. Szasz, Summation of slowly convergent series, J. Math. Physics 29, 272 to 279 (Jan. 1950).

[11] J. Barkley Rosser, Theory and application of $\int_{0}^{z} e^{-x^{2}} d x$ and $\int_{0}^{z} e^{-p^{2} y^{2}} d y \int_{0}^{y} e^{-x^{2}} d x$ (Mapleton House, Brooklyn, N. Y., 1948).

[12] J. Barkley Roser, Explicit remainder terms for some asymptotic series. In press.

Los Angeles, October 31, 1949. 